\title{
Facial Asymmetry Assessment from 3D Shape Sequences - the Clinical Case of Facial Paralysis
}

\author{
Paul Audain Desrosiers ${ }^{1}$, Yasmine Bennis ${ }^{2}$, Boulbaba Ben Amor ${ }^{1}$, Mohamed Daoudi ${ }^{1}$, Pierre \\ Guerreschi $^{2}$ \\ ${ }^{1}$ Telecom Lille, CRIStAL (UMR CNRS 9189) \\ ${ }^{2}$ Service de chirurgie plastique reconstructrice et esthétique, Universite Lille 2 Droit et Santé, Lille, France \\ \{desrosiers, boulbaba.benamor,mohamed.daoudi\}@telecom-lille.fr, yasbennis0311@gmail.com, \\ pierre.guerreschi@chru-lille.fr
}

Keywords: $\quad$ 4D faces, Dense Scalar Fields, face asymmetry, facial paralysis, dynamic faces.

\begin{abstract}
This paper addresses the problem of quantifying the facial asymmetry from dynamic 3D data. We investigate here the role of $4 \mathrm{D}$ (i.e. 3D+time) data to reveal the amount of both static and dynamic asymmetry, in a clinical use-case of facial paralysis. The final goal is to provide tools and solutions to clinicians for facial paralysis assessment and monitoring, which can provide qualitative and quantitative evaluations. To this end, the approach proposed here consider 3D facial sequences and adopt a recently-developed Riemannian approach for facial deformation analysis. After a preprocessing step, each frame of a given 3D sequence is approximated by an indexed collection of elastic radial curves. Riemannian shape analysis of obtained curves and their symmetrical counterparts, both elements of the same shape space, give rise to a feature vector, called Dense Scalar Fields (DSFs). The use of these DSFs reveals the amount of bilateral asymmetry of the face, when conveying expressions. That is, given a 3D frame, it is first reflected with respect to the YZ-plane, then compared to the obtained reflection using the DSFs. To exemplify the use of the proposed approach, a new dataset have been collected (of patients) before and after injecting Botulinum Toxin (BT) in related facial muscles. Experimental results obtained on this dataset show that the proposed approach allows clinicians to evaluate the facial asymmetry before and after the medical treatment.
\end{abstract}

\section{INTRODUCTION}

Human face is the most important non-verbal channel which allows to human beings to communicate with their peers. Its dysfunction may causes discomfort among patients affected by facial paralysis. It occurs in the case of partial or complete loss of functioning of certain facial muscles. They are caused by a lesion of the facial nerve for different reasons acute idiopathic facial paralysis (no cause) called a Bell's palsy, trauma, infection, chronic illness, tumor, etc. One common medical treatment, widely used since 1989 in surgery and aesthetic medicine (Clark and Berris, 1989) (Filipo et al., 2012), is to inject in the muscles of the face low doses of Botulinum Toxin (BT) to handle compensatory over-activity of the healthy muscles. The objective is to reduce the degree of asymmetry in the facial dynamics (i.e the facial temporal evolution) when talking or performing facial expressions. In general, the results of the medical treatments are evaluated subjectively by the clinicians after few weeks of each BT injection, so no automated system exists to assist them conducting their assessment. That is, the clinician ask the patient to convey a specific facial expression or to read a text and record him/her using a camera. The observation of the video (recorded before the BT injection) and the current dynamic allows the clinician to evaluate the results and thus monitoring remaining treatments (BT, surgery, etc.). To our knowledge, no automated tools exist nowadays to allow clinicians quantitative evaluation of the BT injection, in terms of facial dynamics.

\subsection{Prior work}

Analyzing 3D facial sequences is still at the beginning. The recent advances of $3 \mathrm{D} / 4 \mathrm{D}$ sensors based on two main technologies - stereo-photogrammetry and structured-light - opened the doors to develop approaches for analyzing static (3D) and dynamic (4D) shapes. In particular, targeting the face recogni- 
tion or expression classification problems, few groups have recently collected new datasets (Sun and Yin, 2008)(Zhang et al., 2014)(Cosker et al., 2011)(Matuszewski et al., 2012) and have developed first techniques (Sun et al., 2010)(Sandbach et al., 2012)(Ben Amor et al., 2014) for expression recognition from 3D sequences. These approaches have demonstrated the role of 3D dynamic faces analysis to reveal deformations hidden in $2 \mathrm{D}$ videos. In fact, 2D images resulted of the projection of the observed scene into a plane (X-Y) which results in loosing the depth component (Z). Using these 3D acquisition sensors allows to recover the Z-coordinates and thus the use of a the complete shape of the 3D face. Current literature is rich of approaches for shape analysis of 3D and 4D faces. However, only few studies have addressed the fundamental question of - How to quantify the amount of bilateral facial asymmetry from the temporal evolution of 3D faces?

Earlier shape analysis studies are based on the detection and tracking of 2D and 3D facial landmarks in facial sequences. For instance, Al-Anezi et al. (AlAnezi et al., 2013) have proposed a new method for automatic tracking of facial landmarks in $4 \mathrm{D}$ videos. A set of 23 anthropometrics landmarks with no permanent ink are marked on the faces of 32 subjects aged 18 to 35 years. The subjects are asked to perform a facial animation like maximal smile, lip purse, cheek puff. They have concluded that an accurate tracking solution facilitate the analysis of the dynamic motion. Shujaat et al. (Shujaat et al., 2014) have pursued the study conducted in (Al-Anezi et al., 2013) and have developed a new method to quantify dynamic 3D facial animations, in order to characterize the dynamics of 3D lips movement in head, and neck oncology patients before and after lower lip split mandibulotomy. A dataset of 7 subjects aged 42-80 years old is collected. Moreover, a set of nine facials soft tissue landmarks are manually annotated on the first frame of each 3D sequence by an operator, and then tracked automatically. Six landmarks are used to analyze the lip motions, assessing of the magnitude, and the three others to track the effect of the head motion, and to align all the frames into a common reference frame. They measured the feasibility of the change in the magnitude, speed, and motion similarity of facial animation. The results show that the magnitude and the speed difference decreased after the surgical operation for the smile and the lip purse, while motion similarity shows a high score in the case of lip purse animation. Recently, Wei et al. (Quan et al., 2012) proposed a novel approach to analyze the asymmetry of the face on 3D dynamic scan. A data set which comes from the Hi4D-ADSIP database is used, and allow them to make a comparison between stroke patients and healthy individuals. At first sight, in each frame, the nose tip is determined, and a sphere of radius $r=130 \mathrm{~mm}$ is centered at the nose tip for segment of the 3D face. Then, with their proposed method, they extracted the symmetry plan of the $3 \mathrm{D}$ face. Thus, the asymmetry plan can be extracted directly from the symmetry plan by using the original data and the mirrored data obtained by reflection across the symmetry plan. After matching the original 3D face and its mirror with the help of ICP, they calculated the Euclidean distance between each point of the 3D original face and its mirror. From this, they measured the higher asymmetry level of the 3D face which based on the bigger value of the average closest distance. They have concluded that, the proposed method is able to measure the asymmetry of the face, facial dysfunction, and the method is also able to detect minor change in asymmetry which not been observed by the clinician during examination.

\subsection{Methodology and paper organization}

The main goal of this work is to propose a comprehensive and automated pipeline for efficient facial asymmetry evaluation. Using a 3D sensor, several 3D video acquisitions are recorded before and after clinician's intervention. A preprocessing step is then applied to each frame of the 3D videos including - holes filling, smoothing, pose normalization, and nose tip detection. On preprocessed 3D faces a mirror operation with respect to the YZ-plane is applied to produce new meshes (i.e the left half the original face corresponds to the right half of the mirrored face). Shape analysis of the obtained faces (original mesh and its mirror) allows alignment and accurate dense registration, leaded jointly. In particular, using the initial velocity vector along the geodesic path connecting their shapes (Ben Amor et al., 2014), one can quantify the amount of deformations between them. As a result, at each instant of the 3D video, a Dense Scalar Field which reflects the facial asymmetry is computed. Finally, the extension of the previous step to the temporal domain allows quantifying the evolution of facial asymmetry across the video. An experimental illustration of the above-mentioned idea is reported where a new dataset of the patient's face before and after BT injection is recorded.

The rest of the paper is organized as follows. In section 2, we describe the clinical data used in this study which consists of 3D facial sequences of patients recorded before and after the clinician's intervention. The Riemannian shape analysis approach 
and its capability to quantify the amount of facial asymmetry is presented in section 3 . An experimental illustration involving the clinical dataset is discussed in section 4 . In section 5 are drawn some concluding remarks and future investigation.

\section{DATASET DESCRIPTION AND PREPROCESSING}

Due to the novelty of the topic, we have conducted with clinicians the acquisition of patients suffering from facial paralysis. The patients are asked to perform some specific facial expressions in front of the 3D camera. These expressions have been dictated by the clinicians.

\subsection{D data acquisition}

In total five patients with facial paralysis have been invited for two acquisition sessions, one time before the BT injection and a second time after two weeks of the injection. The participants have been asked to convey three different expressions (1) normal smile, (2) forced smile, and (3) raising-up the eyebrows. The average duration of the recorded 3D videos is 4-6 seconds with a total of 60 to 100 3D frames. In our acquisition process, we have used the ARTEC MHT 3D scanner $^{1}$. This single-view 3D scanner allows capturing 3D videos of $15 \mathrm{fps}$ (frame per second), each frame consists of about 5500 vertices, and the 3D resolution is $0.5 \mathrm{~mm}$. To allow an optimal acquisition, the participants are asked to sit in front of the camera at a distance of about $80 \mathrm{~cm}$ to continuously focus on a fixed point. In addition, we have recorded following the same settings, 3D sequences of a healthy face (as a control sample). Figure 1 illustrates a sequence of $3 \mathrm{D}$ facial surfaces obtained using the 3D sensor of a patient's face asked to exhibit a forced smile.

\subsection{Pre-processing of 3D frames}

As mentioned before, the 3D sequences presents imperfections such as missing data (i.e holes in the mesh), noise (i.e. spikes) and contain undesirable parts (neck, clothes, hair, etc.). We applied the following pipeline in order to extract the informative part of the face,

- Clean up the meshes by removing identical vertices,

\footnotetext{
$1_{\text {http: }} / / /$ ww . artec3d.com/fr/hardware/ artec-mht/
}

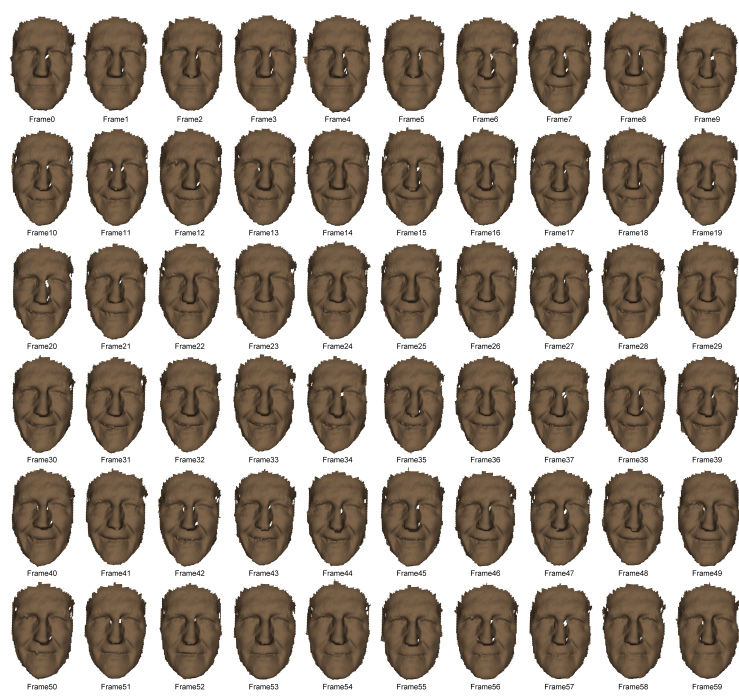

Figure 1: An example of 3D sequence of a patient's face exhibiting a forced (maximum) smile. The sequence follows the evolution model neutral-onset-apex-offset-neutral.

- Fill small holes on the mesh by interpolation,

- Detect the nose tip of each face on each frame,

- Crop the face, the nose tip is used to define a sphere with a constant radius which serves to crop the face region,

- Smooth the meshes using a Laplacian filter to reduce the noise,

- Apply a mirror operator on the 3D faces with respect to the YZ-plan,

- Apply the ICP (Iterative Closest Point) algorithm between the original 3D frame and the mirrored $3 \mathrm{D}$ frame for pose normalization.

An efficient implementation of this pipeline is designed using filters of the $\mathrm{VTK}^{2}$ library. This preprocessing pipeline is applied to each $3 \mathrm{D}$ frame of the 3D video, separately. As a result, we obtain a 3D animations of the 3D faces and their mirrored. The next step is to extract radial curves and to quantify the amount of asymmetry by comparing each 3D shapes to their reflections.

The problem is turned to a comparison of $3 \mathrm{D}$ (static) facial shapes, i.e. at each instant $t$, we preprocessed the 3D face with its mirrored. To achieve such comparison, one needs for an accurate registration algorithm which can handle the non-rigid deformations (bending, stretching/compression) of the facial surfaces. The registration of vertices across 3D faces is an important ingredient when studying their shapes (we assume here an invariance to rigid motions

\footnotetext{
${ }^{2}$ http://www.vtk.org
} 
- translation, scaling and rotation). Specifically, in comparing shapes of faces, it is important that similar biological parts are registered to each other, in particular the left and right halves of the face, when studying the face asymmetry. Several methods have been proposed in the literature as discussed above such as the Non-rigid ICP algorithm (Cheng et al., 2015), the Free Form Deformation (FFD) algorithm (Sandbach et al., 2012) and the Thin-plate Spline (TPS) algorithm (Fang et al., 2012). Most of these solutions try to find an optimal registration between two 3D faces, however, their cost functions which minimize the distance between 3D meshes is not a proper metric; it is not even symmetric. That is, the optimal registration of a 3D face $F^{1}$ to another 3D face $F^{2}$ may not be the same as the registration of $F^{2}$ to $F^{1}$. This makes difficult to interpret the results (i.e. quantifying the divergence between the compared 3D faces). The Riemannian framework used in (Ben Amor et al., 2014) for 4D facial expression analysis grounding on elastic radial curves (i.e. an elastic metric is used (Srivastava et al., 2011)) provides a nice physical interpretation of measuring the facial deformations between curves using a combination of stretching and bending. These elastic deformations are captured by the Dense Scalar Field (DSFs) features. Hence, the main motivation of using a Riemannian approach is to perform registration that matches corresponding anatomical features, and obtain deformation fields that are physically interpretable. In the next section, we recall essential materials to compute the Dense Scalar Fields (DSFs) and illustrates their used to quantify the amount of facial asymmetry. Finally, we will make their extensions to study dynamic 3D faces.

\section{Bilateral Facial Asymmetry}

Given a 3D facial sequence, our aim is to measure the amount of bilateral symmetry at each time and extend it to the temporal dimension. We will start by the first case (i.e. static 3D face), the most common way to quantify the asymmetry is to first detect the plane of symmetry of the face (called also the mirror plane), then apply registration algorithms as the well-know Iterative Closest Point as proposed in (Quan et al., 2012). Here, the most problematic step is to detect accurately the mirror plane before comparing the shape divergence between the two halves of the facial surface. Quan et al. (Quan et al., 2012) consider the 3D vertices of the face as a cloud of points in $\mathbb{R}^{3}$, they apply a Principal Component Analysis (PCA) to extract its dominant direction, which corresponds to the mirror plane. When this approach achieved good results on "normal" faces, it is problematic to use on faces with paralysis. That is, in the latter, the plane location may be far from the the nose tip, for example. A second problem of this approach, is the use of the ICP algorithm, which consider in its cost function only rigid transformations (translation, scaling and rotation) and a very basic optimization criteria based on the pointto-point (or point-to-plane) distance. This makes difficult to achieve any physical interpretation of the results. In the case of facial paralysis, the registration algorithm may register different anatomical parts on the two halves of the $3 \mathrm{D}$ face.

According to the above-mentioned issues, we propose in this paper a different approach to measure the bilateral facial asymmetry, when first avoiding the mirror plane detection, and use a Riemannian shape analysis framework developed recently (Ben Amor et al., 2014) to achieve accurate registration. The basic idea is to approximate the facial surface by an indexed (ordered) collection of radial curves, then consider pairwise curves comparison, using a Riemannian elastic metric which account for the stretching and shrinking of the face. Before reaching this step, we avoid the step of mirror plane detection by applying a simple mirroring to the original face according to the YZ-plane, then align the two surfaces using the ICP algorithm, and finally use the Riemannian approach to quantify the optimal deformation between the 3D face and the mirrored face. To this end, we exploit the notion of geodesic (shortest path between the facial surfaces) on the shape space that we will note $\mathcal{S}^{[0,2 \pi]}$, and we will detail later.

Let first note the facial curves emanating from the nose tip and tracking directions dictated by an angle $\alpha \in[0,2 \pi[$. The obtained collection of curves provides, Figure 2, not only a nice approximation of the face, but also impose on it a parametrization. That is, considering the nose tip as an origin, each point on the face is identified, in a unique way, by $\alpha$ the index of the curve and $k$, the index of the point on the curve. Based on this radial curves representation, the problem of face-to-face registration is turned to a pairwise-curves registration. Elastic shape analysis of open and closed curves is a well-explored problem. An emerging solution using Riemannian geometry have demonstrated convincing results, when the problems of alignment and registration are leaded in the same and unique step. Recently, Ben Amor et al. (Ben Amor et al., 2014) have extended the geometric framework of curves proposed by (Srivastava et al., 2011) to facial surfaces. Using the SRVF representation $q^{\alpha}(t)$ of the facial curves $\beta^{\alpha}(t)$, where $\alpha$ is the curve index on the face, to form the shape space $\mathcal{S}^{[0,2 \pi]}$. As the shape space is a quotient space an 
Hyper-sphere of the Hilbert space $L^{2}\left([0,1], \mathbb{R}^{3}\right)$, the geodesic path between two facial shapes is given by the minor arc of the great circle of the Hyper-sphere.

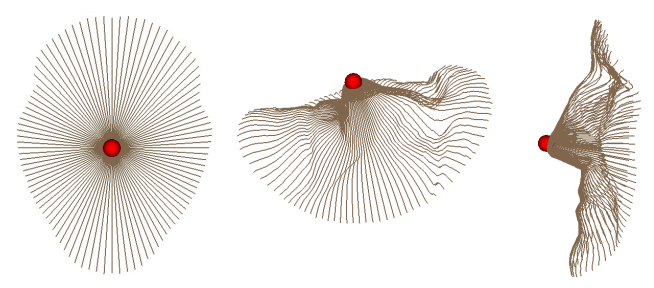

Figure 2: Three views of the same collection of curves extracted to approximate a 3D facial shape. The point in red represent the detected nose tip (origin of the radial curves).

To capture and model the deformations between the 3D face and its mirror during different facial expressions, we pursued with the work of Ben Amor et al. (Ben Amor et al., 2014) which have proposed to represent the facial surface through a set of parameterized radial curves that originate from the tip of the nose. Approximating the facial surface by an ordered set of radial curves, which locally captures its shape can be seen as a parameterization of the facial surface. The mathematical setup for the shape theory offered here comes from Hilbert space analysis.

A facial surface is represented by a collection of radial curves and a Riemannian framework is used to study shapes of these curves. We start by representing facial curves as absolutely continuous maps from $\beta:[0,1] \rightarrow \mathbb{R}^{3}$ and our goal is to analyze shapes represented by these maps. The problem in studying shapes using these maps directly is that they change with re-parameterizations of curves. If $\gamma$ is a re-parameterization function (typically a diffeomorphism from $[0,1]$ to itself), then under the standard $\mathbb{L}^{2}$ norm, the quantity $\left\|\beta_{1}-\beta_{2}\right\| \neq\left\|\beta_{1} \circ \gamma-\beta_{2} \circ \gamma\right\|$, which is problematic. The solution comes from choosing a Riemannian metric under which this inequality becomes equality and the ensuing analysis simplifies. As described in (Srivastava et al., 2011), we represent the facial curves using a new function $q$, called the square-root velocity function (SRVF) (see Eq. (1)). The advantage of using SRVF representation is that under this representation the elastic metric becomes the standard $\mathbb{L}^{2}$ metric and an identical reparameterization of curves preserves the $\mathbb{L}^{2}$ norm of between their SRVFs. The mapping from a curve $\beta$ to $q$ is a bijection (up to a translation) and the space of all SRVFs is the Hilbert space of all square-integrable maps of the type $q:[0,1] \rightarrow \mathbb{R}^{3}$. This space under the natural $\mathbb{L}^{2}$ inner product is actually a vector space and geodesics between points in this space are straight lines.

With the proposed representation, a 3D facial sur- face is approximated by an indexed collection of radial curves $\beta_{\alpha}$, where the index $\alpha$ denotes the angle formed by the curve with respect to a reference radial curve. In particular, the reference radial curve (i.e., the curve with $\alpha=0$ ) is chosen as oriented along the vertical axis, while the other radial curves are separated each other by a fixed angle and are ordered in a clockwise manner.

Considering a generic radial curve $\beta$ of the face, it can be parameterized as $\beta: I \rightarrow \mathbb{R}^{3}$, with $I=[0,1]$, and mathematically represented through the squareroot velocity function (SRVF) (Joshi et al., 2007; Srivastava et al., 2011), denoted by $q(t)$, according to:

$$
q(t)=\frac{\dot{\beta}(t)}{\sqrt{\|\dot{\beta}(t)\|}}, t \in[0,1] .
$$

This specific representation has the advantage of capturing the shape of the curve and makes the calculus simpler. Let us define the space of the SRVFs as $C=\left\{q: I \rightarrow \mathbb{R}^{3},\|q\|=1\right\} \subset \mathbb{L}^{2}\left(I, \mathbb{R}^{3}\right)$, with $\|\cdot\|$ indicating the $\mathbb{L}^{2}$ norm. With the $\mathbb{L}^{2}$ metric on its tangent space, $C$ becomes a Riemannian manifold. Basically, with this parametrization each radial curve is represented on the manifold $C$ by its SRVF. According to this, given the SRVFs $q_{1}$ and $q_{2}$ of two radial curves, the shortest path $\psi^{*}$ on the manifold $C$ between $q_{1}$ and $q_{2}$ (called geodesic path) is a critical point of the following energy function:

$$
E(\psi)=\frac{1}{2} \int\|\dot{\psi}(\tau)\|^{2} d \tau,
$$

where $\psi$ denotes a path on the manifold $C$ between $q_{1}$ and $q_{2}, \tau$ is the parameter for traveling along the path $\psi, \dot{\psi} \in T_{\psi}(C)$ is the tangent vector field on the curve $\psi \in \mathcal{C}$, and $\|$.$\| denotes the \mathbb{L}^{2}$ norm on the tangent space.

Since elements of $\mathcal{C}$ have a unit $\mathbb{L}^{2}$ norm, $\mathcal{C}$ is a hypersphere in the Hilbert space $\mathbb{L}^{2}\left(I, \mathbb{R}^{3}\right)$. As a consequence, the geodesic path between any two points $q_{1}, q_{2} \in C$ is simply given by the minor arc of the great circle connecting them on this hypersphere, $\psi^{*}:[0,1] \rightarrow \mathcal{C}$. This is given by:

$$
\psi^{*}(\tau)=\frac{1}{\sin (\theta)}\left(\sin ((1-\tau) \theta) q_{1}+\sin (\theta \tau) q_{2}\right),
$$

where $\theta=d_{\mathcal{C}}\left(q_{1}, q_{2}\right)=\cos ^{-1}\left(\left\langle q_{1}, q_{2}\right\rangle\right)$. We point out that $\sin (\theta)=0$, if the distance between the two curves is zero, in other words $q_{1}=q_{2}$. In this case, for each $\tau, \psi^{*}(\tau)=q_{1}=q_{2}$.

The tangent vector field on this geodesic is then written as $\frac{d \psi^{*}}{d \tau}:[0,1] \rightarrow T_{\psi}(C)$, and is obtained by the following equation:

$$
\frac{d \psi^{*}}{d \tau}=\frac{-\theta}{\sin (\theta)}\left(\cos ((1-\tau) \theta) q_{1}-\cos (\theta \tau) q_{2}\right) \text {. }
$$


Knowing that on geodesic path, the covariant derivative of its tangent vector field is equal to $0, \frac{d \psi^{*}}{d \tau}$ is parallel along the geodesic $\psi^{*}$ and one can represent it with $\left.\frac{d \psi^{*}}{d \tau}\right|_{\tau=0}$ without any loss of information. Accordingly, Eq. (4) becomes:

$$
\left.\frac{d \psi^{*}}{d \tau}\right|_{\tau=0}=\frac{\theta}{\sin (\theta)}\left(q_{2}-\cos (\theta) q_{1}\right)(\theta \neq 0) .
$$

Based on the above representation, we define a Dense Scalar Field capable to capture deformations between two corresponding radial curves $\beta_{\alpha}^{1}$ and $\beta_{\alpha}^{2}$ of two 3D faces approximated by a collection of radial curves.

Let $q$ and $q^{r}$ the SRVFs of the curves $\beta$ and its reflection $\beta^{r}$, respectively. $\psi^{*}$ is the optimal path (the geodesic) connecting $q$ and $q^{r}$ and the angle $\theta$ is the length of the geodesic which represents the geodesic distance between $q$ and $q^{r}$. By integrating $\theta$ over all $\alpha \in[0,2 \pi]$ gives rise to a scalar value which measures the divergence between the face and its reflection. We will use this measure later on in section 4 in our quantitative analysis. To quantify densely the divergence between faces across a 3D video, Ben Amor et al. (Ben Amor et al., 2014) proposed the Dense Scalar Fields. Roughly speaking, it consists to compute the initial velocity vector $\left.\frac{d \psi^{*}}{d \tau}\right|_{\tau=0}$ along the geodesic $\psi^{*}$, as the geodesic is by definition a constant speed curve on the shape manifold in Eq. (5).

As describe above, the Dense Scalar Field (between a curve and its reflection) denotes the magnitude of $\left.\frac{d \psi^{*}}{d \tau}\right|_{\tau=0}$ at each point of the curves.
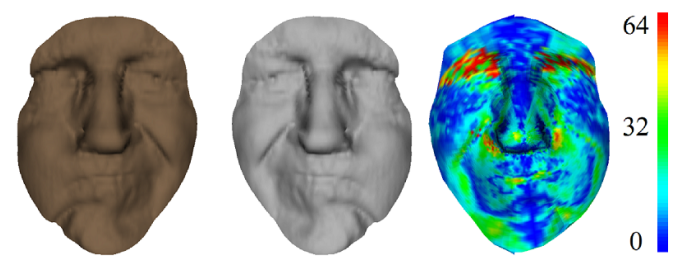

Figure 3: An example of computing the Dense Scalar Fields (DSFs) on a patient's face with paralysis. From left to right (a) original 3D face, (2) 3D face after reflection, and (3) the DSFs shown as a color map mapped on the original 3D face.

Figure 3 illustrates this idea, on the left the original static 3D face, in the middle its reflection and on the right the result of DSFs computation given as a color map of 64 colors ranging from blue to red. In this figure, cold colors represent the most symmetric parts of the 3D face and warm colors highlight the most asymmetric parts. Colors in between reflect a scale of the amount of bilateral asymmetry of the face. Compared to the 3D landmarks-based approach adopted in (Shujaat et al., 2014), our approach provides dense measurements of the bilateral asymmetry on the face. This could be of high importance for clinicians to evaluate locally the bilateral asymmetry of the face. This is because the BT injection is done locally in some specific muscles.

The main motivation behind computing the shape deviation between an arbitrary 3D facial shape and its mirrored shape is to avoid the difficult task of mirror plane detection. In fact, our approach allows to quantify accurately the bilateral (left-right) face asymmetry by (1) a fine alignment of the two shapes using the ICP algorithm, then (2) compute the shape deviation using elastic radial curves (i.e. computing DSFs). Thus, the quantifying the bilateral asymmetry is independent of any plane of symmetry. In particular, this is suitable when working with paralyzed faces when conveying expressions for whom the detection of a plane of symmetry is not an easy task.

\section{Experiments and discussions}

In this section we illustrate experimentally the use of DSFs to quantify the bilateral symmetry of the face. To this end, we used some samples taken from the new dataset described in section 2 . To conduct these experiments, given a 3D sequence of the face, each frame is first reflected then aligned to the original face. As a final step, the DSFs are calculated between the original and the reflected (aligned) shapes. We present in the following four main experiments which involve (1) Neural states pre- and post-operative of a patient's face; (2) Smiling faces (natural) in pre- and post-operative; (3) Forced smiling in pre- and postoperative; and (4) Eyebrows raised in pre- and postoperative. In the following, we report also quantitative and qualitative results on a control subject's face (i.e. normal or healthy subject's face). In these experiments, the facial surfaces are approximated with a total of 100 radial curves, each consists of 50 points, in total each DSF vector contains 5000 scalar points.

1. Neutral State - in this experiment, we simply restrict our study to a static neutral face. Thus, a single 3D surface is considered for the patient's face before and after the BT injection. Figure 4 illustrates the DSFs computed to capture densely the degree of bilateral symmetry on the 3D face. It is quite clear from this figure that only minors changes are shown when comparing the pre- and the post-operative asymmetry results. In contrast, a difference exists when comparing with the asymmetry of a control healthy face. We can note also that the face is not perfectly symmetric (see third row of Figure 4, and the acquisition pro- 
cess may cause some aberrant depth calculations, in particular in the eyes regions. a)

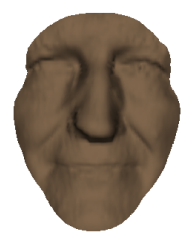

b)
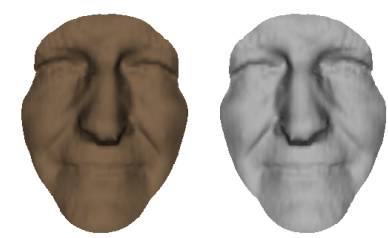

c)
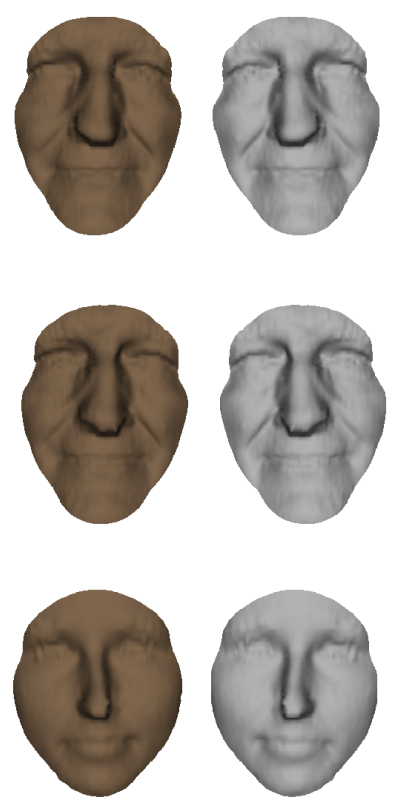
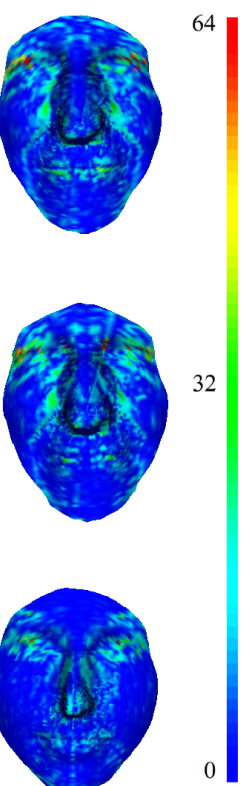
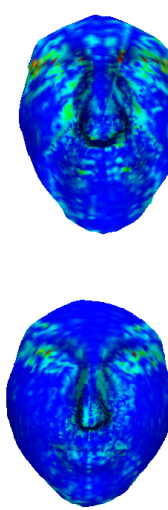

a)
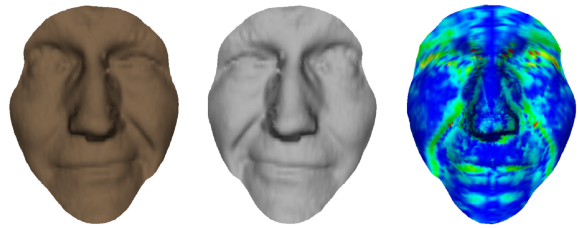

b)
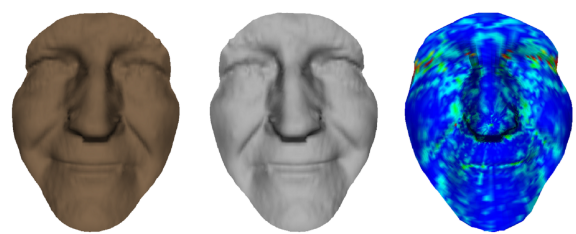

c)
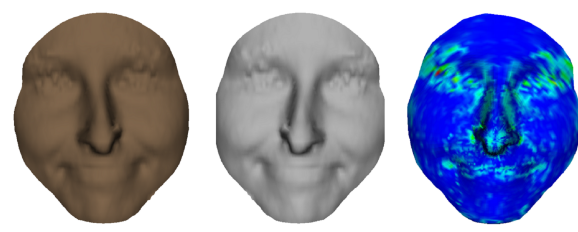

0
Figure 5: Natural smiling experiment (a) pre-operative, (b) post-operative and (c) healthy subject.

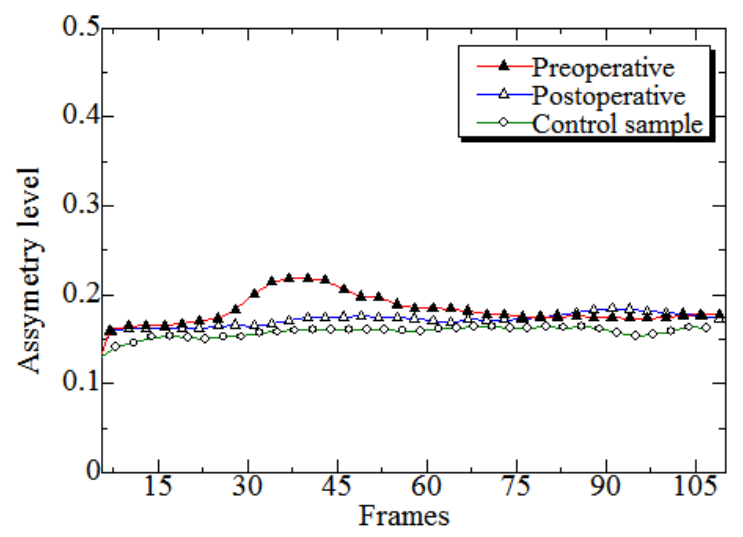

4: From left to right, the original shape, its reflection, and the bilateral asymmetry mapped into the reflection shape. From top to bottom, DSFs on (a) pre-operative, (b) post-operative, and (c) healthy subject's faces.

From this first experiment, its is important that the patient convey a facial expression to allow in-depth analysis of the results.

2. Smiling - in this experiment, the patient is asked to perform a natural smile. In Figure 5 we report the degree of asymmetry for the apex (maximum expression) of the smile. Compared to the previous experiment (Figure 4) the DSFs reveal the difference, in terms of asymmetry, between the pre-operative and the post-operative result. Looking to the first two rows of Figure 5, one can note an improvement of facial symmetry after the BT injection on the same patient. This can be seen in the surface of the blue areas in the two DSFs. It is clear that the amount of asymmetry is reduced along the Zygomaticus muscle. We recall that the post-operative 3D acquisitions happened 2 weeks after the BT injection.

The bilateral asymmetry is evaluated at each frame of the 3D video, we can note here report the DSFs color map along the 3D video, however, we report in Figure 6, the geodesic distance (global deviation value) between the facial shape and its reflection. From this graph, we note a decrease of the facial asymmetry when the expression happened in the temporal segment between the frames 28 and 60 .

This graph showed clearly the importance of considering the temporal evolution of the facial 3D shape
Figure 6: Degree of asymmetry across the video for the natural smiling experiment.

in stead of the static one. When comparing the postoperative results with the healthy face (curves in red and green) in Figure 6, one can note the efficiency of the BT injection to symmetrizing the facial shape and its temporal evolution. However, what happen if the expression is more prominent than a natural smile?

3. Forced smiling - in response of the previous question, the patients are asked to convey a forced (maximum) smile. Then the same pipeline is applied as previously.

From Figure 7, one can establish the same conclusions than in the previous experiment. That is the degree of asymmetry of static and dynamic faces decreases after the BT injection. It can be seen from 
a)
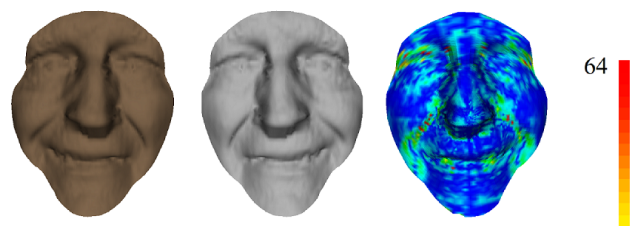

b)
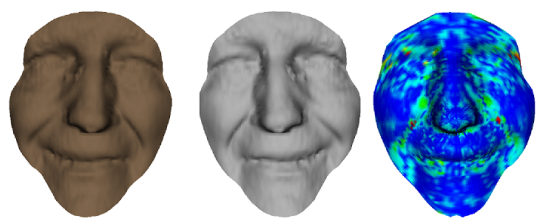

c)
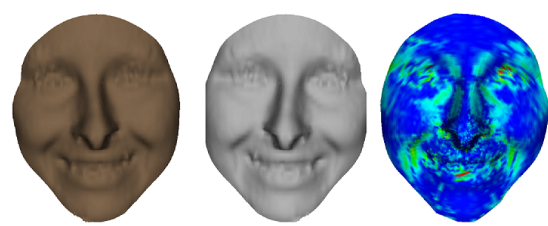

Figure 7: Forced (maximum) smiling experiment.

this graph in Figure 8 that during the time-interval between frames 14 to the end, when the expression happened the amount of bilateral asymmetry before the injection is higher (red curve) than after. The blue curve (after the BT injection) is always higher than the green one (healthy subject). This could be explained by the fact that healthy face taken is our experiments is younger than the patient's face.

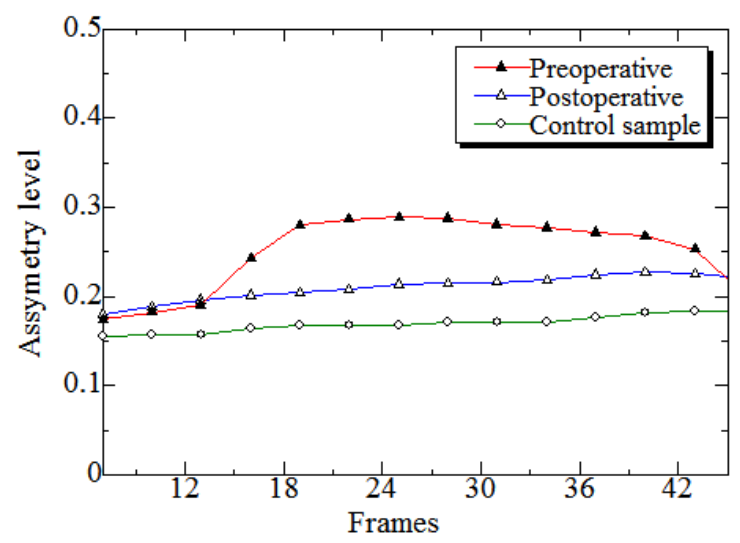

Figure 8: Forced smiling in the experiment.

5. Raising-up the eyebrows - in this experiment, the patients are asked to raised up his eyebrows. From figure 9, one can note again that the amounts of the asymmetry before and after the BT injection are comparable. This is mainly due to the fact that the BT in-

jection is concentrated only on the zygomaticus muscles and the muscles related to the eyebrows movements (i.e. the corrugator muscle) do not received any treatments. This result is also confirmed with the control sample, the amount of asymmetry is huge in the patient's face compared to the normal face.

a)
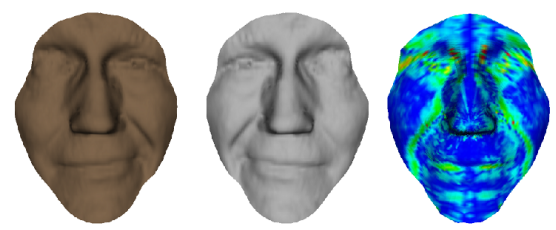

b)
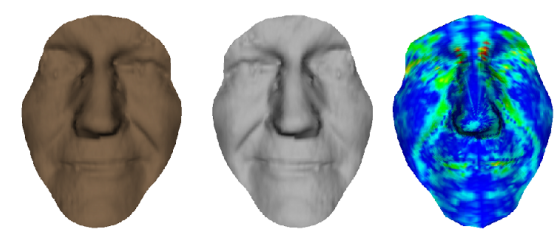

32

c)
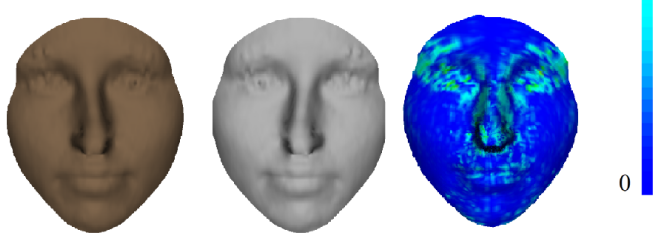

Figure 9: Raising-up the eyebrows experiment.

The graph reported in Figure 10 confirm this observation. We note here a shift in the time interval which corresponds to different starting and ending time of the expression performed before and after receiving the treatments.

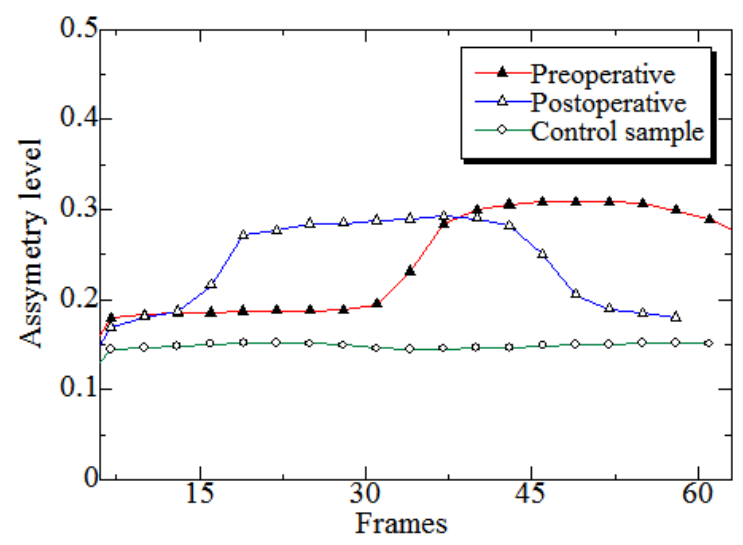

Figure 10: Asymmetry along time for the raising-up the eyebrows experiment.

In the table 1, we present the amount of the asymmetry level measured in each $3 \mathrm{D}$ video sequence. 
Table 1: Maximum asymmetry level in each 3D video.

\begin{tabular}{|l|c|c|c|}
\hline Facial Expression & pre-op & post-op & cs \\
\hline Smiling & 0.218 & 0.173 & 0.159 \\
\hline Force smiling & 0.288 & 0.227 & 0.182 \\
\hline Raise up the eyebrows & 0.308 & 0.291 & 0.146 \\
\hline
\end{tabular}

We can observe that, in the the pre-operative (preop) case, the asymmetry level is higher than the postoperative (post-op) case, and the control sample (cs) has the lower asymmetry level during all facial expressions.

\section{CONCLUSIONS}

In this paper we have proposed a new technique to quantify facial asymmetry from 4D facial data. The main ingredient of this approach is the accommodation of the recently-developed Dense Scalar Fields (Ben Amor et al., 2014) to compare a given face with its reflection and achieve a vertex-to-vertex registration in order to accurately measure the amount of asymmetry in the face. A new dataset of five patients has been collected in clinical conditions. We have demonstrated using the collected 3D dynamic sequences the usefulness of the proposed methodology. In particular, the comparison of the facial asymmetry by using DSF features before and after the BT's injection reveals shows that the proposed approach is a promising solution. This work provides a quantitative tools to the clinicians in order to evaluate the treatment.

\section{REFERENCES}

Al-Anezi, T., Khambay, B., Peng, M., O'Leary, E., Ju, X., and Ayoub, A. (2013). A new method for automatic tracking of facial landmarks in 3d motion captured images (4d). International Journal of Oral and Maxillofacial Surgery, 42(1):9 - 18.

Ben Amor, B., Drira, H., Berretti, S., Daoudi, M., and Srivastava, A. (2014). 4-d facial expression recognition by learning geometric deformations. IEEE T. Cybernetics, 44(12):2443-2457.

Cheng, S., Marras, I., Zafeiriou, S., and Pantic, M. (2015). Active nonrigid ICP algorithm. In 11th IEEE International Conference and Workshops on Automatic Face and Gesture Recognition, FG 2015, Ljubljana, Slovenia, May 4-8, 2015, pages 1-8.

Clark, R. and Berris, C. (1989). Botulinum toxin: a treatment for facial asymmetry caused by facial nerve paralysis. Plast Reconstr Surg, 84(2):353-5.

Cosker, D., Krumhuber, E., and Hilton, A. (2011). A facs valid 3D dynamic action unit database with applica- tions to 3D dynamic morphable facial modeling. In Int. Conf. on Computer Vision (ICCV), pages 22962303.

Fang, T., Zhao, X., Ocegueda, O., Shah, S. K., and Kakadiaris, I. A. (2012). 3d/4d facial expression analysis: An advanced annotated face model approach. Image and Vision Computing, 30(10):738-749.

Filipo, R., Spahiu, I., Covelli, E., Nicastri, M., and Bertoli, G. (2012). Botulinum toxin in the treatment of facial synkinesis and hyperkinesis. The Laryngoscope, 122(2):266-70.

Joshi, S., Klassen, E., Srivastava, A., and Jermyn, I. (2007). A novel representation for Riemannian analysis of elastic curves in $\mathbb{R}^{n}$. In Proc. IEEE Conf. on Computer Vision and Pattern Recognition, pages 10636919, Minneapolis, MN.

Matuszewski, B., Quan, W., Shark, L.-k., McLoughlin, A., Lightbody, C., Emsley, H., and Watkins, C. (2012). Hi4d-adsip 3D dynamic facial articulation database. Image and Vision Computing, 30(10).

Quan, W., Matuszewski, B. J., and Shark, L. (2012). Facial asymmetry analysis based on 3-d dynamic scans. In Proceedings of the IEEE International Conference on Systems, Man, and Cybernetics, SMC 2012, Seoul, Korea (South), October 14-17, 2012, pages 26762681.

Sandbach, G., Zafeiriou, S., Pantic, M., and Rueckert, D. (2012). Recognition of 3D facial expression dynamics. Image and Vision Computing, 30(10):762-773.

Shujaat, S., Khambay, B., Ju, X., Devine, J., McMahon, J., Wales, C., and Ayoub, A. (2014). The clinical application of three-dimensional motion capture (4d): a novel approach to quantify the dynamics of facial animations. International Journal of Oral and Maxillofacial Surgery, 43(7):907 - 916.

Srivastava, A., Klassen, E., Joshi, S. H., and Jermyn, I. H. (2011). Shape analysis of elastic curves in euclidean spaces. IEEE Trans. Pattern Anal. Mach. Intell., 33(7):1415-1428.

Sun, Y., Chen, X., Rosato, M. J., and Yin, L. (2010). Tracking vertex flow and model adaptation for threedimensional spatiotemporal face analysis. IEEE Transactions on Systems, Man, and Cybernetics, Part A, 40(3):461-474.

Sun, Y. and Yin, L. (2008). Facial expression recognition based on 3d dynamic range model sequences. In Proceedings of the 10th European Conference on Computer Vision: Part II, ECCV '08, pages 58-71.

Zhang, X., Yin, L., Cohn, J. F., Canavan, S., Reale, M., Horowitz, A., Liu, P., and Girard, J. M. (2014). Bp4dspontaneous: a high-resolution spontaneous 3D dynamic facial expression database. Image and Vision Computing, 32(10):692 - 706. 